The electro-mechanical integration of the NA62 GigaTracker time tagging pixel detector

This article has been downloaded from IOPscience. Please scroll down to see the full text article.

2010 JINST 5 C12023

(http://iopscience.iop.org/1748-0221/5/12/C12023)

View the table of contents for this issue, or go to the journal homepage for more

Download details:

IP Address: 137.138.124.142

The article was downloaded on 11/07/2011 at 10:57

Please note that terms and conditions apply. 


\title{
The electro-mechanical integration of the NA62 GigaTracker time tagging pixel detector
}

\author{
M. Morel, ${ }^{a, 1}$ A. Kluge,${ }^{a}$ G. Aglieri Rinella, ${ }^{a}$ V. Carassiti, ${ }^{b}$ A. Ceccucci, ${ }^{a}$ J. Daguin,,${ }^{a}$ \\ M. Fiorini, ${ }^{a}$ P. Jarron, ${ }^{c}$ J. Kaplon, ${ }^{a}$ A. Mapelli, ${ }^{d}$ F. Marchetto, ${ }^{c}$ M. Noy, ${ }^{a}$ G. Nuessle,${ }^{d}$ \\ L. Perktold ${ }^{a}{ }^{\text {P. Petagna }}{ }^{a}$ and P. Riedler ${ }^{a}$ \\ ${ }^{a}$ CERN, \\ CH-1211 Geneva 23, Switzerland \\ ${ }^{b}$ INFN Ferrara, \\ Via Saragat 1, 44100 Ferrara, Italy \\ ${ }^{c}$ INFN Torino, \\ Via P. Giurial 1, 10125 Torino, Italy \\ ${ }^{d}$ Universite Catholique de Louvain, \\ 1348 Louvain-la-Neuve, Belgium \\ ${ }^{e}$ EPFL Lausanne, \\ CH-1015 Lausanne, Switzerland \\ E-mail: michel.morel@cern.ch
}

\begin{abstract}
The NA62 GigaTracker is a low mass time tagging hybrid pixel detector operating in a beam with a particle rate of $750 \mathrm{MHz}$. It consists of three stations with a sensor size of $60 \times 27 \mathrm{~mm}^{2}$ containing 18000 pixels, each $300 \times 300 \mu \mathrm{m}^{2}$. The active area is connected to a matrix of $2 \times 5$ pixel ASICs, which time tag the arrival of the particles with a binning of 100 ps. The detector operates in vacuum at -20 to $0^{\circ} \mathrm{C}$ and the material budget per station must be below $0.5 \% \mathrm{X}_{0}$. Due to the high radiation environment of $2 \times 10^{14} 1 \mathrm{MeV}$ neutron equivalent $\mathrm{cm}^{-2} / \mathrm{yr}^{-1}$ it is planned to exchange the detector modules regularly. The low material budget, cooling requirements and the request for easy module access has driven the electro-mechanical integration of the GigaTracker, which is presented in this paper.
\end{abstract}

KEYWORDS: Overall mechanics design (support structures and materials, vibration analysis etc); Detector cooling and thermo-stabilization

\footnotetext{
${ }^{1}$ Corresponding author.
} 


\section{Contents}

1 Introduction 1

2 GTK assembly carrier PCB 1

3 Electro-mechanical integration $\quad 2$

4 Electrical connections 3

5 Cooling options 4

5.1 Micro-channel cooling 4

5.2 GTK gas cooling 4

6 Conclusions 5

\section{Introduction}

The GigaTracker (GTK) is a low mass time tagging hybrid pixel detector composed of three p-in-n pixel sensors in the rare kaon decay experiment, NA62, at the CERN SPS. It is a spectrometer that provides precise measurements of momentum, time and angle of incoming $75 \mathrm{GeV} / \mathrm{c}$ kaon beam. The GTK is composed of three stations (GTK1, GTK2 and GTK3) mounted around four bending magnets as shown in figure 1. Each GTK is placed along the beam line inside a dedicated vacuum vessel.

\section{GTK assembly carrier PCB}

The GTK assembly carrier is a printed circuit board (PCB) with mechanical support and alignment with the beam. The electro-mechanical integration is based on the principle that one GTK module, the assembly and its services, are an integral and compact component, which can be inserted and removed with minimal intervention.
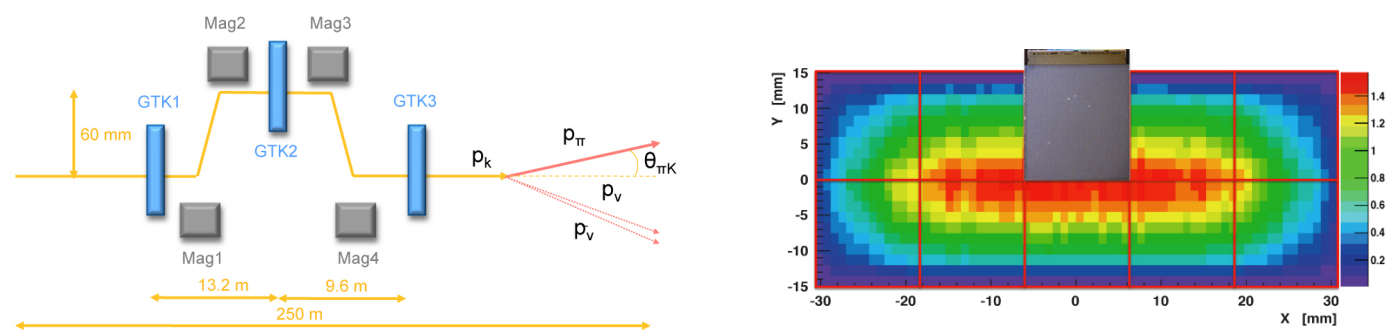

Figure 1. Layout of the GTK stations and the decay. Figure 2. Beam intensity and ASIC configuration. 

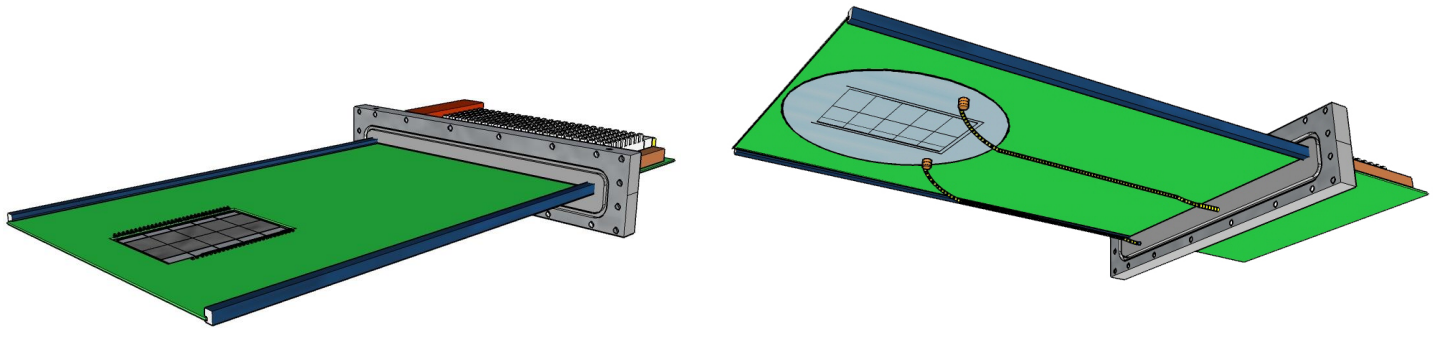

Figure 4. Bottom view of the GTK assembly carrier

Figure 3. Top view of the GTK assembly carrier.

equipped with micro channel cooling.

The assembly carrier is constructed using a mixture of FR4 and Kapton ${ }^{\circledR}$ materials based on sequential build up (SBU) technology. The SBU provides micro vias of $80 \mu \mathrm{m}$ allowing the high signal density requirements close to the ASICs to be accommodated.

The GTK assembly is electrically connected with bonding wires to the GTK assembly carrier, which connects the ASICs to high/low voltage power, to the serial control, status and data interfaces of each chip using dedicated signal traces. It carries optical components outside the vacuum vessel; see figure 3 and figure 4 . The assembly carrier also acts as a vacuum feed through. A flange with an O-ring is tightly glued around the PCB and screwed to the vacuum vessel.

Mechanical guides assure precise alignment of the assembly carrier and thus the silicon assembly with the vessel. Figure 5

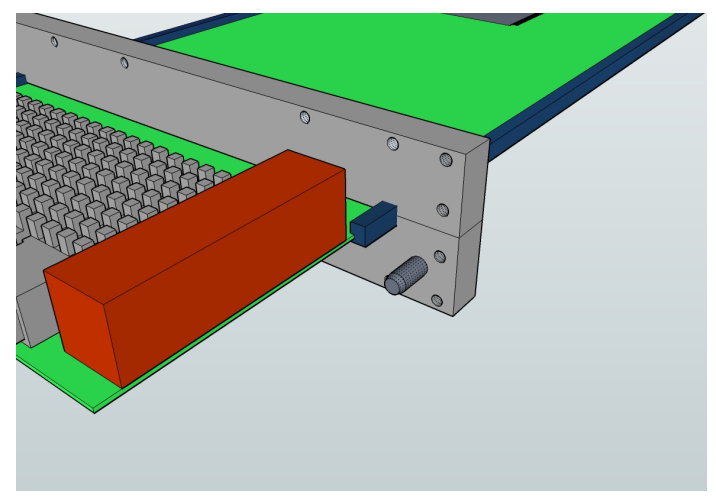

Figure 5. Picture representing details on flange, guides and pin positioning. shows the vacuum feed-though and the positioning guide.

\section{Electro-mechanical integration}

Each of the three GTK stations will be installed inside a dedicated vacuum vessel at three different positions along the NA62 experiment beam line. The vessels for GTK1 and GTK2 are identical and are $292 \mathrm{~mm} \times 239 \mathrm{~mm} \times 190 \mathrm{~mm}$ in size, shown in figure 6 , whereas GTK3 is $550 \mathrm{~mm} \times 560 \mathrm{~mm} \times 2000 \mathrm{~mm}$ as it contains the CHANTI detector planes as well, see figure 7 . The vessel for GTK3 and the CHANTI will be fixed in place whereas the GTK1 and 2 vessels will be mounted on an xy-table for automated alignment to GTK3 and the beam center. Connections with the beam pipe will be made using bellows allowing the vessels to move. 


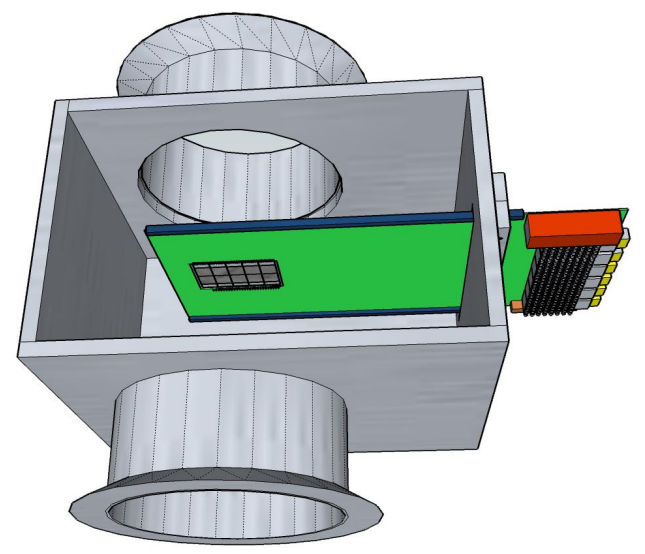

Figure 6. Vacuum vessel design for stations 1 and 2.

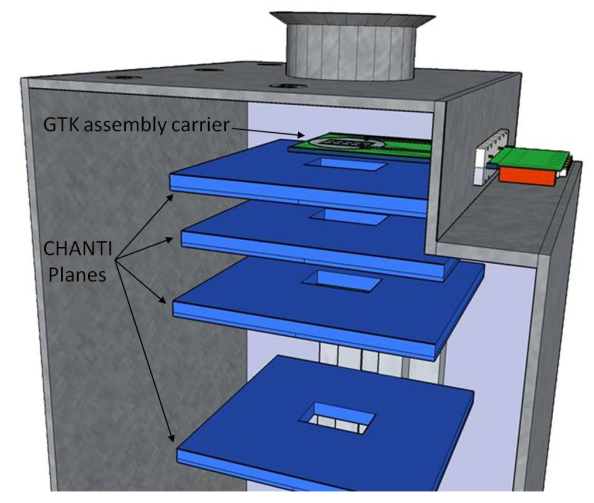

Figure 7. Vacuum vessel design for station 3, showing the CHANTI planes and the GTK assembly carrier.

\section{Electrical connections}

Wire bonds provide the electrical connections between the ASIC wire bond pads, the sensor bias connections and the GTK assembly carrier. Each ASIC requires three different power supplies $1.2 \mathrm{~V}$ digital, $1.2 \mathrm{~V}$ analog and $2.5 \mathrm{~V}$. In order to minimize the electrical interference, each ASIC will be powered individually with separate power supplies. Measurements will show whether the analog and digital supply of an individual chip can be combined on the assembly carrier. For the optical components and clock distribution buffers the card is connected to $3.3 \mathrm{~V}$. This means a total of up to 31 power supply connections for each GTK interface board are re-

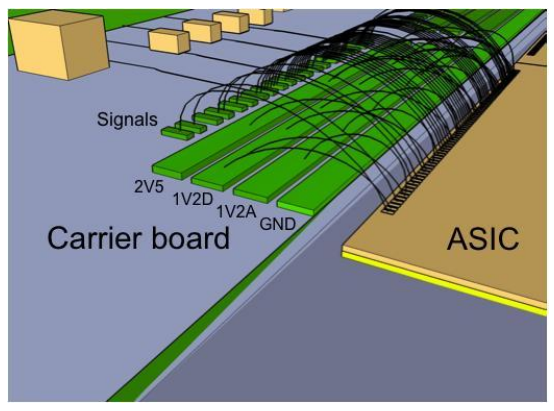

Figure 8. Bonding connections scheme. quired. In order to avoid the need of a precise pitch adapter for the wire bond connection to the PCB, a staggered wire bonding scheme is proposed where the pitch on the PCB does not need to match the ASIC pitch of $73 \mu \mathrm{m}$.

Figure 8 illustrates the principle where the power connections and the signal connections go to different rows on the PCB. Each of the 10 ASICs sends $6 \mathrm{~Gb} / \mathrm{s}$ data on differential lines to the optical components on the assembly carrier. The TDCs inside the ASICs require a low jitter clock signal. The electrical layout of the PCB is driven by these two constraints and requires a study and simulation of signal integrity and special attention to the

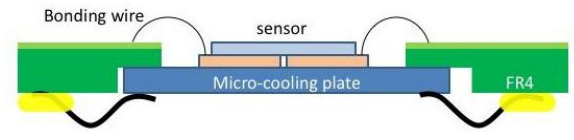
routing. Prototype GTK assembly carriers are going to be produced in order to optimize this layout.

Figure 9 shows a cross section of the module. The FR4 acts as a rigidifier as well as a carrier for the PCB. Due to high variation of temperature in the cooled operation mode and in the uncooled stand-by mode, the silicon components are not glued to the GTK assembly carrier, but are 


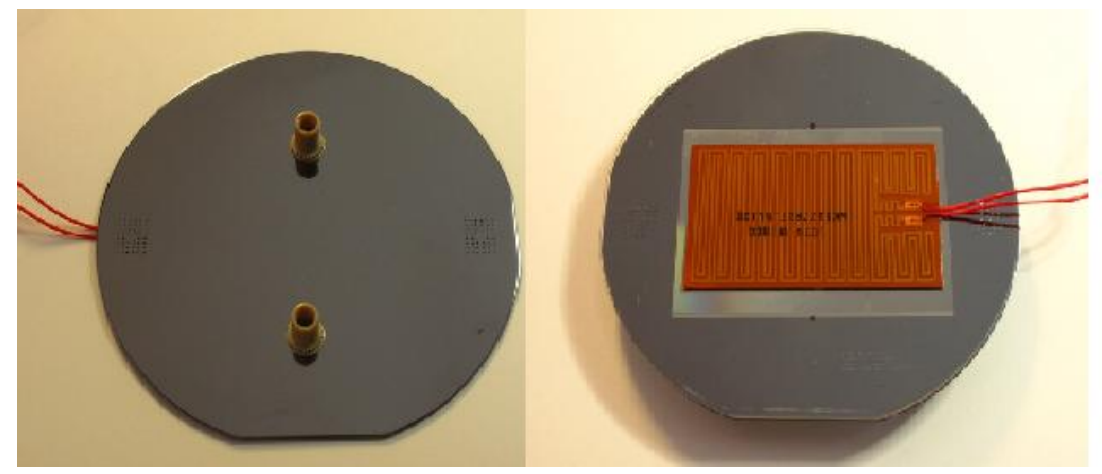

Figure 10. Micro channel plate cooling prototype showing coolant connections (left) and heating foil (right). The left image shows the cooling wafer with two Nanoport connectors and the right image the cooling wafer with a heating foil used to emulate the electronic heat load.

placed in a precisely machined opening and held by carbon fiber clips to maintain the position. This arrangement will avoid mechanical stress due to the different thermal expansion coefficients of silicon and FR4.

\section{Cooling options}

Due to the low mass of the detector module, thermal run-away and destruction needs to be avoided. Furthermore the cooling system for the Gigatracker reduces the radiation damage of the sensor and consequently increases the module life time. Although the upper limit of the operation temperature has been set to $5^{\circ} \mathrm{C}$, a lower operation temperature is desirable. The material budget and the operation in vacuum are the main integration challenges of the project. Mechanics and cooling integration can profit from the fact that no material budget restrictions exist for the area $10 \mathrm{~mm}$ outside the beam. At the time of writing, the working group is pursuing two independent options: 1) a micro-channel structure; 2 ) a gas vessel with Kapton ${ }^{\circledR}$ windows.

\subsection{Micro-channel cooling}

The micro-channel cooling uses a $150 \mu \mathrm{m}$ thick silicon cooling plate containing $120 \times 90 \mu \mathrm{m}^{2}$ micro channels with $C_{6} F_{14}$ as the cooling fluid. The cooling plate is then glued to the sensor assembly. The development aims for an operating temperature of $-20^{\circ} \mathrm{C}$ to $-30^{\circ} \mathrm{C}$. The prototype cooling plate is illustrated in figure 10 .

\subsection{GTK gas cooling}

The gas cooling consists of a vessel with Kapton ${ }^{\circledR}$ windows on both sides of the detector placed inside the beam vacuum where gaseous nitrogen flow provides the cooling. The nitrogen will enter the vessel at a temperature of $100 \mathrm{~K}$ by one side evacuating the dissipated heat by the other side.

The cold fluid will keep the operation temperature of the module less than $5^{\circ} \mathrm{C}$. Figure 11 shows on the left the GTK carrier board equipped with the gas cooling vessel and on the right the picture of the gas vessel prototype. 


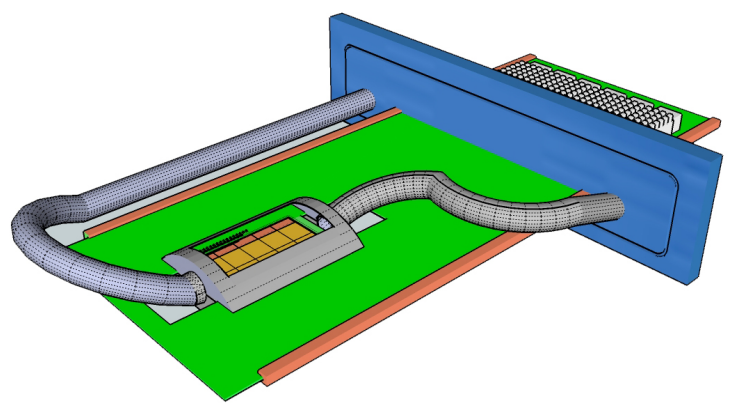

(a) GTK carrier board equipped with gas cooling vessel

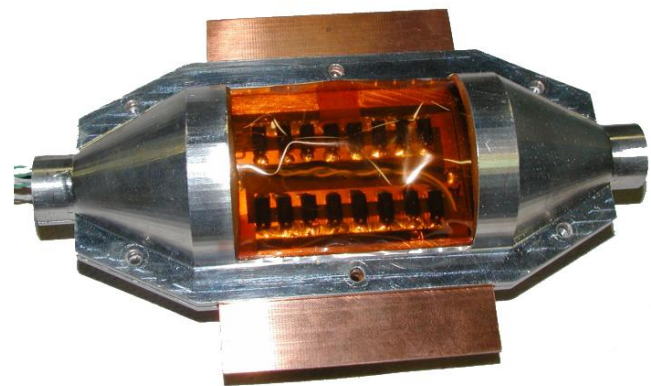

(b) The gas cooling vessel prototype

Figure 11. The Gas Cooling option showing (a) the evisaged integration, and (b) the current prototype.

\section{Conclusions}

The electro-mechanical integration of the GTK stations has been designed allowing fast module interchange with automatic mechanical alignment. Two cooling options are under investigation to find the most suitable.

\section{References}

[1] G. Anelli et al., Proposal to measure the rare decay $K^{+} \rightarrow \pi^{+} v \bar{v}$ at the CERN SPS, CERN-SPSC-2005-013.

[2] A. Kluge et al., NA62 Gigatracker, in Proceedings of 19th International Workshop on Vertex Detectors 2010.

[3] V. Carassiti, GTK cooling vessel prototype, private communication.

[4] A. Mapelli, Micro-channel cooling: status report, private communication. 